Article

\title{
A Novel 3 $\alpha$-p-Nitrobenzoylmultiflora-7:9(11)-diene-29-benzoate and Two New Triterpenoids from the Seeds of Zucchini (Cucurbita pepo L)
}

\section{Reiko Tanaka *, Takashi Kikuchi, Saori Nakasuji, Yasuhiro Ue, Daisuke Shuto, Keishi Igarashi, Rina Okada and Takeshi Yamada}

Laboratory of Medicinal Chemistry, Osaka University of Pharmaceutical Sciences, 4-20-1 Nasahara, Takatsuki, Osaka 569-1094, Japan

* Author to whom correspondence should be addressed; E-Mail: tanakar@gly.oups.ac.jp; Tel./Fax: +81-72-690-1084.

Received: 6 June 2013; in revised form: 21 June 2013 / Accepted: 21 June 2013 /

Published: 26 June 2013

\begin{abstract}
Three novel multiflorane-type triterpenoids, $3 \alpha-p$-nitrobenzoylmultiflora7:9(11)-diene-29-benzoate (1), 3 $\alpha$-acetoxymultiflora-7:9(11)-diene-29-benzoate (2), and $3 \alpha$-acetoxymultiflora-5(6):7:9(11)-triene-29-benzoate (3), along with two known related compounds $\mathbf{4}$ and $\mathbf{5}$ were isolated from the seeds of zucchini (Cucurbita pepo L). Their structures were determined on the basis of 1D and 2D NMR spectroscopy and HREIMS. Triterpenoids possessing a nitro group were not isolated previously.
\end{abstract}

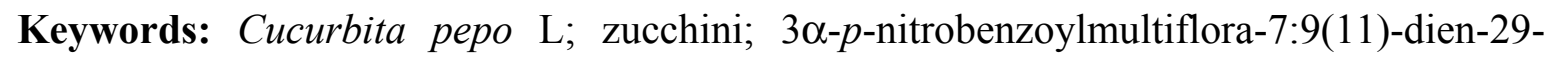
benzoate; 3 $\alpha$-acetoxymultiflora-7:9(11)-diene-29-benzoate; $3 \alpha$-acetoxymultiflora-5(6):7:9 (11)-triene-29-benzoate

\section{Introduction}

The species Cucurbita pepo is a cultivated plant of the genus Cucurbita that includes varieties of squash, gourd, and pumpkin. Cucurbita pepo L (zucchini, also known as field pumpkin or summer squash) (Cucurbitaceae) are widely cultivated in America, Europe, and Asia. The zucchini is a hybrid of the cucumber, and has been a commercially important crop in many countries since the 1950-1960s. It is a highly nutritional low caloric food that requires relatively little effort to prepare. It is full of nutrients like vitamin A, vitamin $\mathrm{C}$, potassium, folate and fiber-all of which support a healthy 
metabolism. Zucchini, grows well in warm climates. This readily available vegetable can also be an important part of weight loss efforts.

As to triterpenoids from Cucurbita pepo L, Appendino reported $3 \alpha$ - $p$-aminobenzoyl, 29-benzoylmultiflor-8-en-7 $\beta$-ol, and 3 $\alpha$-p-aminobenzoylmultiflora-7:9(11)-dien-29-benzoate [1,2]. Barker reported large-scale isolation of bryonolic acid (3ß-hydroxymultiflor-8-en-29-oic acid) [3].Wang reported cucurbitacin glycoside [4], hexanorcucurbitane glycosides [5], and purine-containing cucurbitane triterpenoids [6], extracted from Cucurbita pepo cv dayangua. Ding et al., reported cerebroside, 13(18)-oleanen-3-ol, $\beta$-daucosterol, $\beta$-sitosterol, stigmasterol, dotriacontyl stearate, and tritriacontane from Cucurbita pepo cv dayangua [7]. Shibuya reported the biosynthesis of sterols and triterpenes in higher plants: Panax ginseng, Olea europaea, Taraxacum officinale, Betula platyphylla, Glycyrrhiza glabra, Luffa cylindrica, Pisum sativum, Allium macrostemon, and Cucurbita pepo [8]. Shibuya et al., also reported that three oxidosqualene cyclas (OSC) cDNAs (CPX, CPQ, CPR) were cloned from seedlings of Cucurbita pepo by a homology-based PCR method [9]. Careful examination of the seeds of Cucurbita pepo L. has led to the isolation of three novel multiflorane triterpenoids 1-3 along with the known compounds 4 and $\mathbf{5}$. The structures of 1-3 were determined on the basis of NMR spectroscopy, including $1 \mathrm{D}$ and $2 \mathrm{D}\left({ }^{1} \mathrm{H},{ }^{1} \mathrm{H}-\mathrm{COSY}\right.$, NOESY, HSQC, HMBC) NMR, and EIMS.

\section{Results and Discussion}

The seeds of Cucurbita pepo L. were extracted with $\mathrm{MeOH}$ and the extract was partitioned $\mathrm{Et}_{2} \mathrm{O}$ and $\mathrm{H}_{2} \mathrm{O}$. The $\mathrm{Et}_{2} \mathrm{O}$ soluble portion (216.1 g) was subjected to silica gel column chromatography, medium-pressure liquid chromatography (MPLC), and normal-phase high performance liquid chromatography (HPLC) to yield five triterpenoids 1-5 (Figure 1) Compounds $\mathbf{4}$ and $\mathbf{5}$ were identified

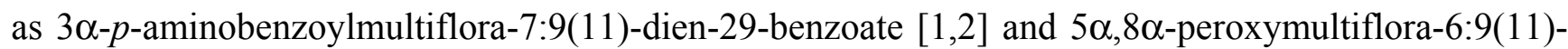
diene-3 $\alpha, 29$-dibenzoate [10], respectively by comparison of their characterization data with literature data.

Figure 1. Structures for compounds 1-5.

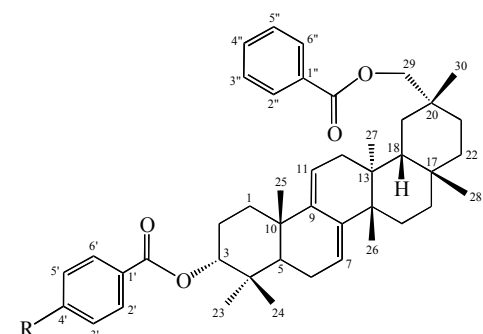

1: $\mathrm{R}=\mathrm{NO}_{2}$

4: $\mathrm{R}=\mathrm{NH}_{2}$

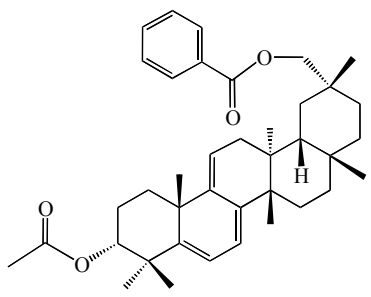

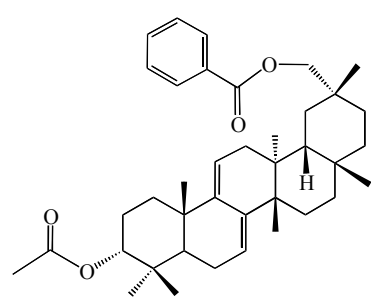

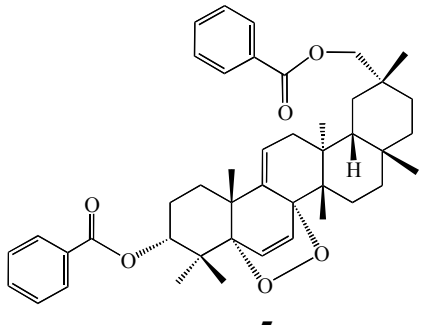

5 
The molecular formula of 1 was determined as $\mathrm{C}_{44} \mathrm{H}_{55} \mathrm{NO}_{6}\left(\mathrm{M}^{+} ; m / z\right.$ 693.4024) based on HREIMS. The UV spectrum showed a heteroannular diene moiety $\left(\lambda_{\max } 230,237,248 \mathrm{~nm}, \log \varepsilon 3.85,3.80\right.$, 3.63). The IR spectrum showed bands assignable to ester groups $\left(v_{\max } 1713,1287 \mathrm{~cm}^{-1}\right)$ and a nitro group ( $v_{\max } 1527,1341 \mathrm{~cm}^{-1}$ ). The ${ }^{1} \mathrm{H}$ - and ${ }^{13} \mathrm{C}$-NMR spectra (Table 1) exhibited signals assignable to seven tertiary methyls, ten $\mathrm{CH}_{2}$ groups including an oxymethylene [ $\delta_{\mathrm{H}} 4.11$ and 4.17 (each $\left.1 \mathrm{H}, \mathrm{d}\right)$ ], three $s p^{3}$ methine groups including an oxymethine $\left[\delta_{\mathrm{H}} 4.97(1 \mathrm{H}, \mathrm{t})\right]$, two trisubstituted olefin $\left[\delta_{\mathrm{H}} 5.24\right.$ $(1 \mathrm{H}$, brd $) ; 5.53(1 \mathrm{H}, \mathrm{brs})]$, six $s p^{3}$ quaternary carbons, a benzoyl group $\left[\delta_{\mathrm{H}} 7.43(2 \mathrm{H}, \mathrm{tt}), 7.52(1 \mathrm{H}, \mathrm{tt})\right.$, $8.08(2 \mathrm{H}, \mathrm{dd}) ; \delta_{\mathrm{C}} 128.6$ (C-3", C-5"), 129.7 (C-2", C-6"), 130.8 (C-1"), 133.0 (C-4"), 166.9 (OCO)] and a $p$-nitrobenzoyl group $\left[\delta_{\mathrm{H}} 8.10(2 \mathrm{H}, \mathrm{dt}), 8.20(2 \mathrm{H}, \mathrm{dt}) ; \delta_{\mathrm{C}} 123.8\left(\mathrm{C}-3^{\prime}, \mathrm{C}-5^{\prime}\right), 130.7\left(\mathrm{C}-2^{\prime}, \mathrm{C}-6^{\prime}\right)\right.$, $\left.136.3\left(\mathrm{C}-1^{\prime}\right), 150.6\left(\mathrm{C}-4^{\prime}\right), 164.4,(\mathrm{OCO})\right]$. In the HMBC spectrum (Figure 2), long-range correlations were observed between Me-23 and C-3 $\left(\delta_{\mathrm{C}} 80.7\right), \mathrm{C}-4, \mathrm{C}-5$, and C-24; between Me-24 and C-3, C-4, C-5, and C-23; between Me-25 and C-1, C-5, C-9 $\left(\delta_{\mathrm{C}} 144.6\right)$, and C-10; between Me-26 and C-8 $\left(\delta_{\mathrm{C}}\right.$ 142.3), C-13, C-14, and C-15; between Me-27 and C-12, C-13, C-14, and C-18; between Me-28 and $\mathrm{C}-16, \mathrm{C}-17, \mathrm{C}-18$, and C-22; between Me-30 and C-19, C-20, C-21, and C-29 $\left(\delta_{\mathrm{C}} 73.0\right)$; between $\mathrm{H}_{2}-29$ and C-19, C-20, C-21, and C-30; between $\mathrm{H}-2^{\prime}$ and $\mathrm{H}_{-} 6^{\prime}\left[\delta_{\mathrm{H}} 8.10(2 \mathrm{H})\right]$ and $\mathrm{C}-4^{\prime}\left(\delta_{\mathrm{C}} 150.6\right)$, $\mathrm{O}-\underline{\mathrm{C}}=\mathrm{O}\left(\delta_{\mathrm{C}} 164.4\right)$; and between $\mathrm{H}-2^{\prime \prime}$ and $\mathrm{H}-6^{\prime \prime}\left[\delta_{\mathrm{H}} 8.08(2 \mathrm{H})\right]$ and $\mathrm{O}-\underline{\mathrm{C}}=\mathrm{O}\left(\delta_{\mathrm{C}} 166.9\right)$. In the ${ }^{1} \mathrm{H}-{ }^{1} \mathrm{H}$ COSY spectrum $\left(\mathrm{H}-7-\mathrm{H}_{2}-6\right.$; H-11- $\mathrm{H}_{2}-12 ; \mathrm{H}-3-\mathrm{H}_{2}-2 ; \mathrm{H}-3^{\prime}$ and $\mathrm{H}-5^{\prime}-\mathrm{H}-2^{\prime}$ and H-6'; H-3" and H-5"-H2", H-6" and H-4") of 1 revealed the partial structures shown in bold face in Figure 2. EIMS showed a molecular ion peak at $\mathrm{m} / \mathrm{z} 693$ which is 30 mass units bigger than that of 4 . Furthermore, the same base ion peak was observed in compounds 1 and 4 at $\mathrm{m} / \mathrm{z} 526$ [M-p-nitrobenzoic acid in 1; M- $p$ aminobenzoic acid in 4]. On the basis of the above spectral data, 1 was established to be a novel $3 \alpha-p$ nitrobenzoylmultiflora-7:9(11)-dien-29-benzoate. Selected NOESY correlations for $\mathbf{1}$ are shown in Figure 3. The configuration of the $p$-nitrobenzoyl group at C-3 was established as the $\alpha$ (axial)orientation due to the NOE correlations between H-3 and Me-23 and Me-24, and the coupling constants of $\mathrm{H}-3\left[\delta_{\mathrm{H}} 4.97\left(\mathrm{t}, J_{3 \beta .2 \alpha ; 3 \beta, 2 \beta}=2.7 \mathrm{~Hz}\right)\right]$. The benzoyl group was at $\mathrm{C}-29$ because the NOESY correlation was observed between $\mathrm{H}_{2}-29$ and $\mathrm{H}-22 \alpha$ and Me-27. Therefore, 1 was determined as $3 \alpha-p-$ nitrobenzoylmultiflora-7:9(11)-dien-29-benzoate. Although, natural products containing nitro groups have been isolated from plants [11], e.g., monocyclic aromatic compounds [12], multicyclic aromatic compounds [13], amino acids and peptides [14], carbohydrates [15], aliphatic compounds [16], and $O$-nitro and $N$-nitro compounds [17], compound $\mathbf{1}$ is the first example which has a nitro group in triterpenoids.

Table 1. ${ }^{1} \mathrm{H}-(500 \mathrm{MHz})$ and ${ }^{13} \mathrm{C}-\mathrm{NMR}(125 \mathrm{MHz})$ spectroscopic data of compounds $\mathbf{1}-\mathbf{3}\left(\mathrm{CDCl}_{3}\right){ }^{\mathrm{a}}$.

\begin{tabular}{|c|c|c|c|c|c|c|c|c|c|c|c|}
\hline \multirow{3}{*}{$\frac{\text { Position }}{1 \alpha}$} & \multicolumn{4}{|c|}{1} & \multicolumn{4}{|c|}{2} & \multicolumn{3}{|c|}{3} \\
\hline & \multicolumn{2}{|c|}{$\delta_{\mathrm{H}}(J$ in $\mathrm{Hz})$} & \multicolumn{2}{|c|}{$\delta_{\mathrm{C}}$} & \multicolumn{2}{|c|}{$\delta_{\mathrm{H}}(J$ in $\mathrm{Hz})$} & \multicolumn{2}{|c|}{$\delta_{\mathrm{C}}$} & \multicolumn{2}{|r|}{$\delta_{\mathrm{H}}(J$ in $\mathrm{Hz})$} & $\delta_{\mathrm{C}}$ \\
\hline & 1.78 & $\mathrm{~m}$ & 30.8 & $(t)$ & 1.67 & $\mathrm{~m}$ & 30.2 & $(t)$ & 1.76 & $\operatorname{td}(14.0,4.3)$ & $30.3 \quad(t)$ \\
\hline $1 \beta$ & 1.62 & $\mathrm{~m}$ & & & 1.48 & $\mathrm{~m}$ & & & 1.72 & $\mathrm{~m}$ & \\
\hline $2 \alpha$ & 1.92 & $\mathrm{~m}$ & 23.3 & $(t)$ & 1.73 & $\mathrm{~m}$ & 23.0 & $(t)$ & 1.82 & $\mathrm{~m}$ & $22.8 \quad(t)$ \\
\hline $2 \beta$ & 2.07 & $\mathrm{~m}$ & & & 1.92 & $\mathrm{~m}$ & & & 2.06 & $\mathrm{~m}$ & \\
\hline 3 & 4.97 & $\mathrm{t}(2.7)$ & 80.7 & (d) & 4.67 & $\mathrm{t}(2.7)$ & 78.2 & (d) & 4.75 & $\mathrm{dd}(3.5,2.4)(3.0)$ & $77.4 \quad(d)$ \\
\hline 4 & & & 37.4 & $(s)$ & & & 36.1 & $(s)$ & & & $38.9 \quad(s)$ \\
\hline
\end{tabular}


Table 1. Cont.

\begin{tabular}{|c|c|c|c|c|c|c|c|c|c|c|c|c|}
\hline \multirow{3}{*}{$\begin{array}{c}\text { Position } \\
5\end{array}$} & \multicolumn{4}{|c|}{1} & \multicolumn{4}{|c|}{2} & \multicolumn{4}{|c|}{3} \\
\hline & \multicolumn{2}{|c|}{$\delta_{\mathrm{H}}(J$ in $\mathrm{Hz})$} & \multicolumn{2}{|c|}{$\delta_{\mathrm{C}}$} & \multicolumn{2}{|c|}{$\delta_{\mathrm{H}}(J$ in $\mathrm{Hz})$} & \multicolumn{2}{|l|}{$\delta_{\mathrm{C}}$} & \multicolumn{2}{|c|}{$\delta_{\mathrm{H}}(J$ in $\mathrm{Hz})$} & \multicolumn{2}{|l|}{$\delta_{\mathrm{C}}$} \\
\hline & 1.77 & $\mathrm{~m}$ & 43.8 & $(d)$ & 1.63 & $\mathrm{~d}(4.9)$ & 42.8 & (d) & & & 148.9 & $(s)$ \\
\hline $6 \alpha$ & 2.21 & $\mathrm{~m}$ & 24.0 & $(t)$ & 2.12 & $\operatorname{brt}(4.9)$ & 23.7 & $(t)$ & 5.85 & $\mathrm{~d}(6.4)$ & 118.1 & (d) \\
\hline $6 \beta$ & 2.10 & $\mathrm{~m}$ & & & 2.02 & $\mathrm{~m}$ & & & & & & \\
\hline 7 & 5.53 & brs & 118.3 & $(d)$ & 5.49 & brs & 118.1 & $(d)$ & 5.61 & $\mathrm{~d}(6.4)$ & 114.4 & (d) \\
\hline 8 & & & 142.3 & $(s)$ & & & 141.8 & $(s)$ & & & 141.2 & $(s)$ \\
\hline 9 & & & 144.6 & $(s)$ & & & 144.3 & $(s)$ & & & 144.6 & $(s)$ \\
\hline 10 & & & 36.4 & $(s)$ & & & 36.1 & $(s)$ & & & 39.2 & $(s)$ \\
\hline 11 & 5.24 & brd (4.5) & 114.3 & $(d)$ & 5.20 & $\mathrm{~d}(5.3)$ & 113.8 & $(d)$ & 5.34 & $\mathrm{dt}(5.0,2.1)$ & 118.4 & (d) \\
\hline $12 \alpha$ & 2.10 & $\mathrm{~m}$ & 39.6 & $(t)$ & 2.07 & $\mathrm{~m}$ & 39.3 & $(t)$ & 2.16 & $\mathrm{dd}(17.4,6.2)$ & 40.1 & $(t)$ \\
\hline $12 \beta$ & 1.77 & $\begin{array}{l}\text { dd (11.7, } \\
4.8)\end{array}$ & & & 1.75 & $\mathrm{~m}$ & & & 1.83 & $\mathrm{~m}$ & & \\
\hline 13 & & & 37.5 & $(s)$ & & & 37.3 & $(s)$ & & & 38.2 & $(s)$ \\
\hline 14 & & & 40.3 & $(s)$ & & & 40.0 & $(s)$ & & & 40.0 & $(s)$ \\
\hline $15 \alpha$ & 1.85 & $\mathrm{~m}$ & 27.6 & $(t)$ & 1.71 & $\mathrm{~m}$ & 27.3 & $(t)$ & 1.77 & $\mathrm{~m}$ & 26.8 & $(t)$ \\
\hline $15 \beta$ & 1.42 & $\mathrm{~m}$ & & & 1.37 & $\mathrm{~m}$ & & & 1.36 & $\mathrm{~m}$ & & \\
\hline $16 \alpha$ & 1.69 & $\mathrm{~m}$ & 36.8 & $(t)$ & 1.70 & $\mathrm{~m}$ & 36.6 & $(t)$ & 1.74 & $\mathrm{~m}$ & 36.6 & $(t)$ \\
\hline $16 \beta$ & 1.52 & $\mathrm{~m}$ & & & 1.50 & $\mathrm{t}(3.8)$ & & & 1.52 & $\mathrm{dt}(10.0,3.1)$ & & \\
\hline 17 & & & 31.8 & $(s)$ & & & 31.5 & $(s)$ & & & 31.6 & $(s)$ \\
\hline 18 & 1.65 & $\mathrm{~m}$ & 44.8 & $(d)$ & 1.65 & $\mathrm{~m}$ & 44.6 & $(d)$ & 1.70 & $\mathrm{dd}(9.2,2.6)$ & 44.6 & (d) \\
\hline $19 \alpha$ & 1.81 & $\mathrm{~m}$ & 28.6 & $(t)$ & 1.82 & $\mathrm{~m}$ & 28.3 & $(t)$ & 1.84 & $\mathrm{~m}$ & 27.9 & $(t)$ \\
\hline $19 \beta$ & 1.61 & $\mathrm{~m}$ & & & 1.54 & $\mathrm{~m}$ & & & 1.55 & $\mathrm{~m}$ & & \\
\hline 20 & & & 31.9 & $(s)$ & & & 31.6 & $(s)$ & & & 31.6 & $(s)$ \\
\hline $21 \alpha$ & 1.48 & $2 \mathrm{H}, \mathrm{m}$ & 30.2 & $(t)$ & 1.58 & $2 \mathrm{H}, \mathrm{m}$ & 30.0 & $(t)$ & 1.46 & $\mathrm{~m}$ & 30.1 & $(t)$ \\
\hline $21 \beta$ & & & & & & & & & 1.60 & $\mathrm{~m}$ & & \\
\hline $22 \alpha$ & 1.79 & $\mathrm{~m}$ & 34.4 & $(t)$ & 1.80 & $\mathrm{dd}(10.1,4.4)$ & 34.0 & $(t)$ & 1.81 & $\mathrm{~m}$ & 34.0 & $(t)$ \\
\hline $22 \beta$ & 0.94 & $\mathrm{~m}$ & & & 0.94 & $\mathrm{~m}$ & & & 0.95 & $\mathrm{dt}(13.8,3.1)$ & & \\
\hline 23 & 0.92 & $\mathrm{~s}$ & 27.7 & $(q)$ & 0.84 & $\mathrm{~s}$ & 22.0 & $(q)$ & 1.08 & $\mathrm{~s}$ & 26.8 & $(q)$ \\
\hline 24 & 1.07 & $\mathrm{~s}$ & 22.2 & $(q)$ & 0.98 & $\mathrm{~s}$ & 27.2 & $(q)$ & 1.22 & $\mathrm{~s}$ & 31.6 & $(q)$ \\
\hline 25 & 0.99 & $\mathrm{~s}$ & 20.7 & $(q)$ & 0.93 & $\mathrm{~s}$ & 20.4 & $(q)$ & 1.17 & $\mathrm{~s}$ & 30.7 & $(q)$ \\
\hline 26 & 0.95 & $\mathrm{~s}$ & 22.1 & $(q)$ & 0.93 & $\mathrm{~s}$ & 21.7 & $(q)$ & 1.04 & $\mathrm{~s}$ & 21.0 & $(q)$ \\
\hline 27 & 0.91 & $\mathrm{~s}$ & 19.7 & $(q)$ & 0.88 & $\mathrm{~s}$ & 19.6 & $(q)$ & 0.83 & $\mathrm{~s}$ & 19.8 & $(q)$ \\
\hline 28 & 1.13 & $\mathrm{~s}$ & 31.3 & $(q)$ & 1.12 & $\mathrm{~s}$ & 31.0 & $(q)$ & 1.13 & $\mathrm{~s}$ & 31.1 & $(q)$ \\
\hline $29 \mathrm{~A}$ & 4.11 & $\mathrm{~d}(10.7)$ & 73.0 & $(t)$ & 4.10 & $\mathrm{~d}(10.7)$ & 72.8 & $(t)$ & 4.08 & $\mathrm{~d}(10.9)$ & 72.6 & $(t)$ \\
\hline $29 \mathrm{~B}$ & 4.17 & $\mathrm{~d}(10.7)$ & & & 4.15 & $\mathrm{~d}(10.7)$ & & & 4.16 & $\mathrm{~d}(10.9)$ & & \\
\hline 30 & 1.12 & $\mathrm{~s}$ & 30.6 & $(q)$ & 1.11 & $\mathrm{~s}$ & 30.5 & $(q)$ & 1.11 & $\mathrm{~s}$ & 30.7 & $(q)$ \\
\hline $3-\mathrm{OCO}$ & & & 164.4 & $(s)$ & & & 170.9 & $(s)$ & & & 171.0 & $(s)$ \\
\hline $1^{\prime}$ & & & 136.3 & $(s)$ & 2.03 & $\mathrm{~s}$ & 21.3 & (q) & 2.00 & $\mathrm{~s}$ & 21.3 & $(s)$ \\
\hline
\end{tabular}


Table 1. Cont.

\begin{tabular}{|c|c|c|c|c|c|c|c|c|c|c|c|c|}
\hline \multirow{2}{*}{ Position } & \multicolumn{4}{|c|}{1} & \multicolumn{4}{|c|}{2} & \multicolumn{4}{|c|}{3} \\
\hline & \multicolumn{2}{|r|}{$\delta_{\mathrm{H}}(J$ in $\mathrm{Hz})$} & \multicolumn{2}{|l|}{$\delta_{\mathrm{C}}$} & \multicolumn{2}{|c|}{$\delta_{\mathrm{H}}(J$ in $\mathrm{Hz})$} & \multicolumn{2}{|c|}{$\delta_{\mathrm{C}}$} & \multicolumn{2}{|c|}{$\delta_{\mathrm{H}}(J$ in $\mathrm{Hz})$} & \multicolumn{2}{|l|}{$\delta_{\mathrm{C}}$} \\
\hline $2^{\prime}, 6^{\prime}$ & 8.10 & $\mathrm{dt}(8.9,2.1)$ & 130.7 & (d) & & & & & & & & \\
\hline $3^{\prime}, 5^{\prime}$ & 8.20 & $\mathrm{dt}(8.9,2.1)$ & 123.8 & (d) & & & & & & & & \\
\hline $4^{\prime}$ & & & 150.6 & $(s)$ & & & & & & & & \\
\hline 29-OCCO & & & 166.9 & $(s)$ & & & 166.7 & $(s)$ & & & 166.7 & $(s)$ \\
\hline $1 "$ & & & 130.8 & $(s)$ & & & 130.6 & $(s)$ & & & 130.6 & $(s)$ \\
\hline 2", 6" & 8.08 & $2 \mathrm{H}, \mathrm{dd}(7.4,2.1)$ & 129.7 & (d) & 8.08 & $\begin{array}{l}2 \mathrm{H}, \mathrm{dd} \\
(7.4,1.4)\end{array}$ & 129.4 & (d) & 8.07 & $\begin{array}{l}2 \mathrm{H}, \mathrm{dd} \\
(8.2,1.2)\end{array}$ & 129.5 & (d) \\
\hline 3", 5" & 7.43 & $\begin{array}{l}2 \mathrm{H}, \mathrm{tt} \\
(7.4,2.1)\end{array}$ & 128.6 & (d) & 7.45 & $\begin{array}{l}2 \mathrm{H}, \mathrm{tt} \\
(7.4,1.4)\end{array}$ & 128.4 & (d) & 7.45 & $\begin{array}{l}2 \mathrm{H}, \mathrm{tt} \\
(8.2,1.2)\end{array}$ & 128.4 & (d) \\
\hline 4" & 7.52 & $\mathrm{tt}(7.4,2.1)$ & 133.0 & (d) & 7.58 & $\mathrm{tt}(7.4,1.4)$ & 132.8 & (d) & 7.56 & $\mathrm{tt}(8.2,1.2)$ & 132.8 & $(d)$ \\
\hline
\end{tabular}

${ }^{a}$ Assignments were based on ${ }^{1} \mathrm{H}^{1}{ }^{1} \mathrm{H}$ COSY, HMQC, HMBC and NOESY spectroscopic data.

Figure 2. Selected ${ }^{1} \mathrm{H}-{ }^{1} \mathrm{H}$ COSY and HMBC correlations for $\mathbf{1}$.

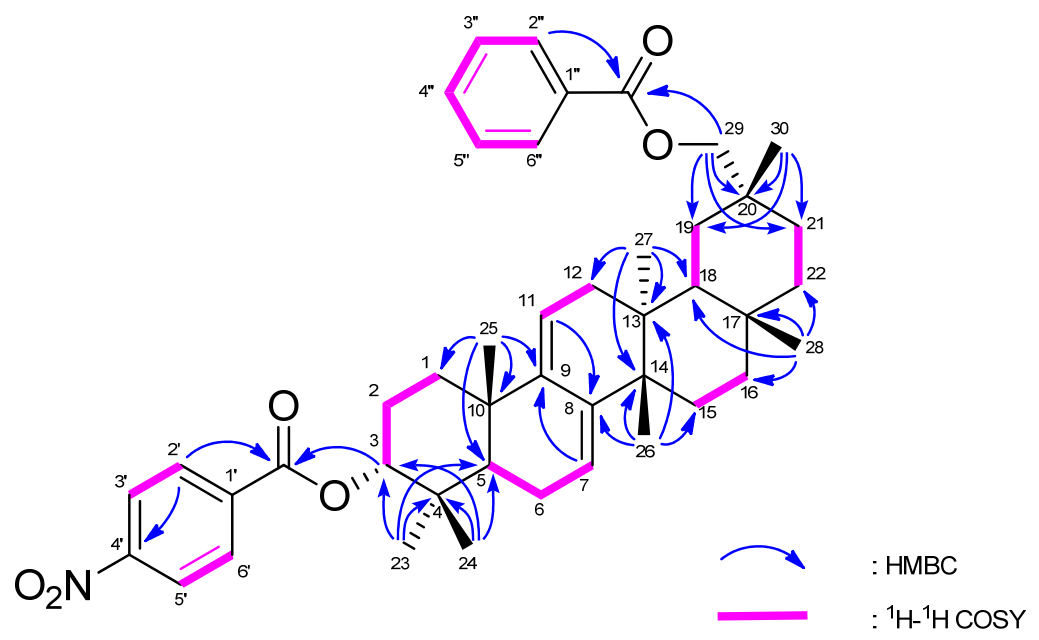

Figure 3. Key NOE correlations for $\mathbf{1}$.

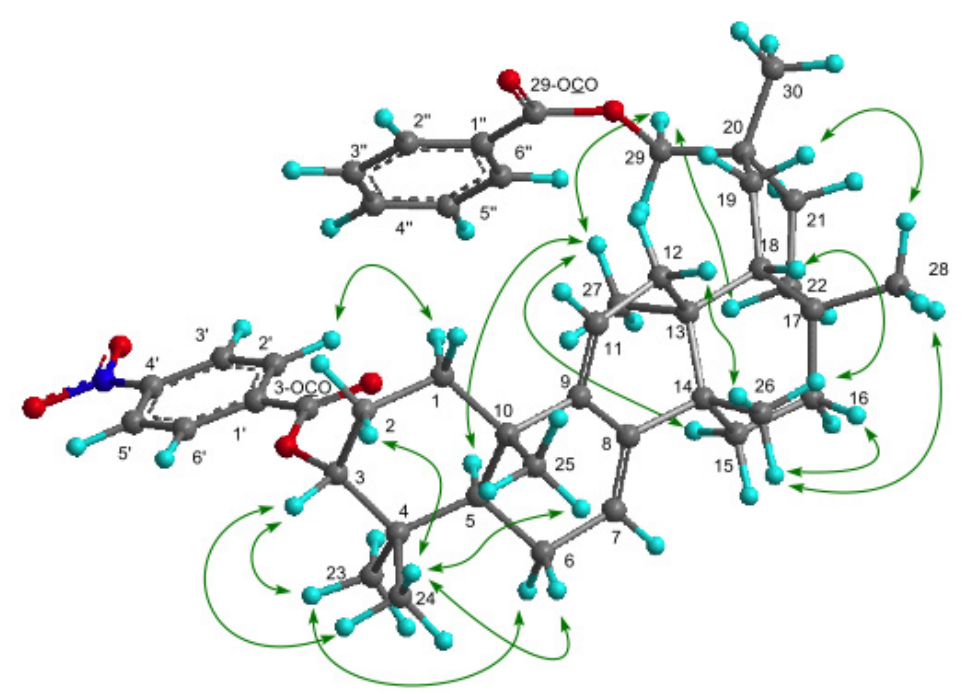


Compound 2 was assigned the molecular formula $\mathrm{C}_{39} \mathrm{H}_{54} \mathrm{O}_{4}\left(\mathrm{M}^{+} ; m / z\right.$ 586.4016) based on HREIMS. The UV absorption band showed a heteroannular diene $\left(\lambda_{\max } 222,237 \mathrm{~nm}, \log \varepsilon 3.93,3.95\right)$. The IR spectrum showed the presence of ester groups $\left(v_{\max } 1743,1718,1271 \mathrm{~cm}^{-1}\right)$. The ${ }^{1} \mathrm{H}-$ and ${ }^{13} \mathrm{C}-\mathrm{NMR}$ spectra (Table 1) exhibited signals assignable to seven tertiary methyls, ten $\mathrm{CH}_{2}$ groups including an oxymethylene $\left[\delta_{\mathrm{H}} 4.10,4.15\right.$ (each $\left.\left.1 \mathrm{H}, \mathrm{d}\right)\right]$, three $s p^{3}$ methine groups including an oxymethine $\left[\delta_{\mathrm{H}} 4.67\right.$ $(1 \mathrm{H}, \mathrm{t})], \Delta 7,9(11)$-diene $\left(\delta_{\mathrm{H}} 5.20,5.49\right)$, an acetyl group $\left[\delta_{\mathrm{H}} 2.03(3 \mathrm{H}, \mathrm{s})\right]$, and a benzoyl group [ $\delta_{\mathrm{H}}$ $7.45(2 \mathrm{H}, \mathrm{tt}), 7.58(1 \mathrm{H}, \mathrm{tt}), 8.08(2 \mathrm{H}, \mathrm{dd}) ; \delta_{\mathrm{C}} 128.4$ (C-3", C-5"), 129.4 (C-2", C-6"), $130.6\left(\mathrm{C}-1^{\prime \prime}\right)$, 132.8 (C-4"), 166.7 (OCO)]. In the HMBC spectrum of 2 (Figure 4), long-range correlations were observed between Me-25 $\left(\delta_{\mathrm{H}} 0.93\right)$ and C-9 $\left(\delta_{\mathrm{C}} 144.3\right)$; between Me-26 $\left(\delta_{\mathrm{H}} 0.93\right)$ and C-8 $\left(\delta_{\mathrm{C}} 141.8\right)$; between Me-23 $\left(\delta_{\mathrm{H}} 0.84\right)$ and Me-24 $\left(\delta_{\mathrm{H}} 0.98\right)$ and C-3 $\left(\delta_{\mathrm{C}} 78.2\right)$; and between Me-30 $\left(\delta_{\mathrm{H}} 1.11\right)$ and C-29 [ $\left.\delta_{\mathrm{C}} 72.8(\mathrm{t})\right]$. The spectral data indicated 2 to be a novel $3 \alpha$-acetoxymultiflora-7:9(11)-diene29-benzoate.

Figure 4. Selected ${ }^{1} \mathrm{H}-{ }^{1} \mathrm{H}$ COSY and $\mathrm{HMBC}$ correlations for 2.

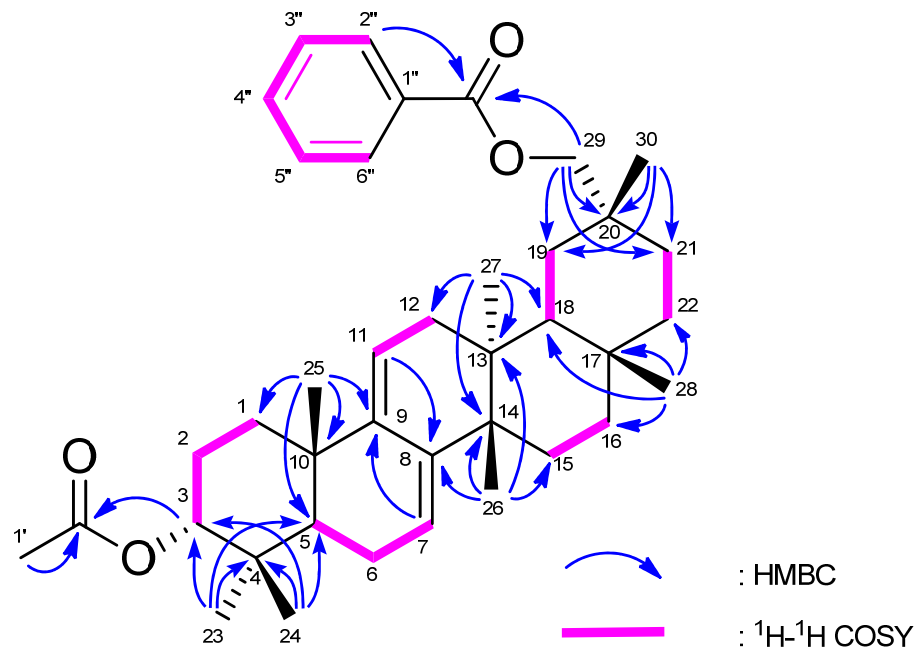

The molecular formula of 3 was determined as $\mathrm{C}_{39} \mathrm{H}_{52} \mathrm{O}_{4}\left(\mathrm{M}^{+} ; m / z\right.$ 584.3864) based on HREIMS. The UV spectrum showed a 5(6),7,9(11)-triene moiety $\left(\lambda_{\max } 227,304,315,334 \mathrm{~nm}, \log \varepsilon\right.$ 4.19, 3.98, 4.00, 3.72). The IR spectrum showed bands assignable to ester groups $\left(v_{\max } 1725,1239 \mathrm{~cm}^{-1}\right)$. The ${ }^{1} \mathrm{H}$ - and ${ }^{13} \mathrm{C}-\mathrm{NMR}$ spectra (Table 1 ) exhibited signals due to seven tertiary methyls, nine $\mathrm{CH}_{2}$ groups including an oxymethylene $\left[\delta_{\mathrm{H}} 4.08,4.16\right.$ (each $\left.\left.1 \mathrm{H}, \mathrm{d}\right)\right]$, three $s p^{3}$ methine groups including an oxymethine $\left[\delta_{\mathrm{H}} 4.75(1 \mathrm{H}, \mathrm{t})\right]$, three trisubstituted olefin $\left[\delta_{\mathrm{H}} 5.34(1 \mathrm{H}, \mathrm{dt}) ; 5.61(1 \mathrm{H}, \mathrm{d}) ; 5.85(1 \mathrm{H}, \mathrm{d})\right]$, six $s p^{3}$ quaternary carbons, an acetyl group $\left[\delta_{\mathrm{H}} 2.00 ; \delta_{\mathrm{C}} 171.0(\mathrm{~s})\right]$, and a benzoyl group $\left[\delta_{\mathrm{H}} 7.45(2 \mathrm{H}\right.$, tt), $7.56(1 \mathrm{H}, \mathrm{tt}), 8.07(2 \mathrm{H}, \mathrm{dd}) ; \delta_{\mathrm{C}} 128.4$ (C-3", C-5"), 129.5 (C-2", C-6"), 130.6 (C-1"), 132.8 (C-4"), $166.7(\mathrm{OCO})]$. In the $\mathrm{HMBC}$ spectrum (Figure 5), long-range correlations were observed between Me-23 $(\delta 1.08)$ and C-3 $\left(\delta_{\mathrm{C}} 77.4\right), \mathrm{C}-4, \mathrm{C}-5\left[\delta_{\mathrm{C}} 148.9(\mathrm{~s})\right]$, and C-24; between Me-24 $\left(\delta_{\mathrm{H}} 1.22\right)$ and C$3, \mathrm{C}-4, \mathrm{C}-5$, and C-23; and between Me-25 ( $\delta$ 1.17) and C-1, C-5, C-9 [ $\left.\delta_{\mathrm{C}} 144.6(\mathrm{~s})\right]$, and C-10. In the ${ }^{1} \mathrm{H}-{ }^{1} \mathrm{H}$ COSY spectrum, H-6 $\left(\delta_{\mathrm{H}} 5.85\right)$ correlated with only H-7 $\left(\delta_{\mathrm{H}} 5.61\right)$; H-11 $\left(\delta_{\mathrm{H}} 5.34\right)$ correlated with $\mathrm{H}_{2}-12\left(\delta_{\mathrm{H}} 1.83,2.16\right)$. EIMS showed a fragment ion peak at $m / z 524[\mathrm{M}-\mathrm{AcOH}]^{+}$as a base ion peak. Based on the spectral data, the structure of $\mathbf{3}$ was established as $3 \alpha$-acetoxymultiflora5(6):7:9(11)-trien-29-benzoate. 
Figure 5. Selected ${ }^{1} \mathrm{H}-{ }^{1} \mathrm{H}$ COSY and HMBC correlations for 3.

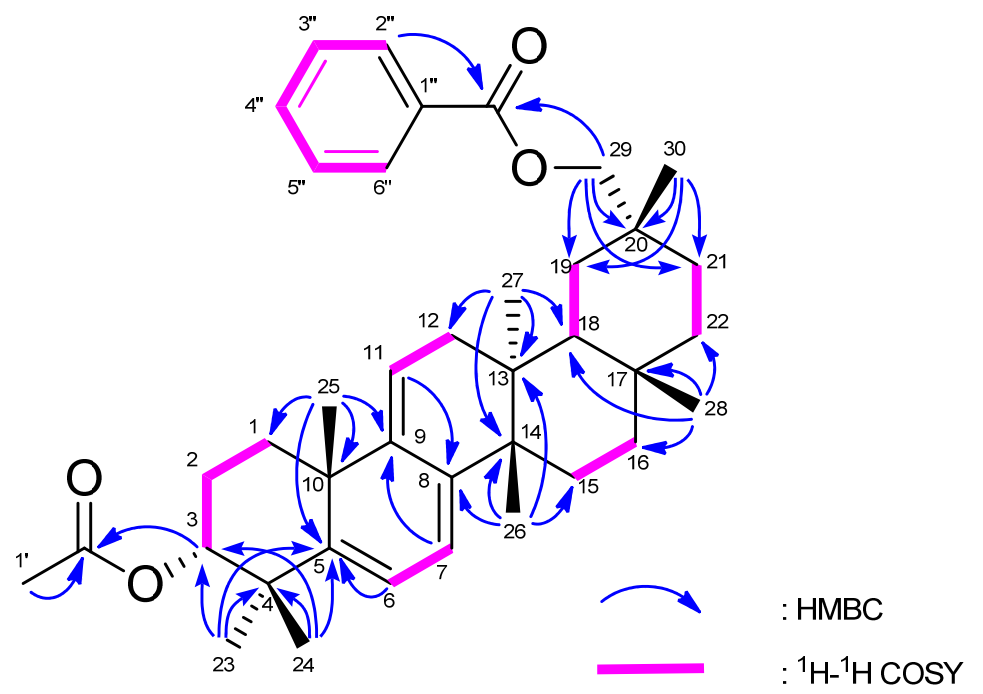

Compounds 1-5 were evaluated for cytotoxic activity against HL-60 and P388 cells using MTT methods (Table 2) [18]. Although, 2 exhibited weak cytotoxic activity against HL-60 (IC $5025.7 \mu \mathrm{M})$ and $\mathrm{P} 388\left(\mathrm{IC}_{50} 75.1 \mu \mathrm{M}\right), \mathbf{1}$ and $\mathbf{3}-\mathbf{5}$ showed no activity against either cell line. Compound $\mathbf{3}$ showed melanogenesis inhibitory activity with low cytotoxicity at $100 \mu \mathrm{M}$ (melanin content $66.9 \%$, cell viability 92.5\%) (Table 3). Compound 2 exhibited strong melanogenesis inhibitory activity, although probably due to its cytotoxic action (cell viability $32.8 \%, 69.3 \%$, and $87.6 \%$ at 100,30 , and $10 \mu \mathrm{M}$, respectively).

Table 2. Cytotoxic activity of multiflorane-type triterpenes from Cucurbita pepo seeds.

\begin{tabular}{|c|c|c|}
\hline \multirow[b]{2}{*}{ Compound } & \multicolumn{2}{|c|}{$I_{50}(\mu M)^{a}$} \\
\hline & $\begin{array}{c}\text { HL-60 } \\
\text { (human leukemia) }\end{array}$ & $\begin{array}{c}\text { P388 } \\
\text { (murine leukemia) }\end{array}$ \\
\hline 1 & $>100$ & $>100$ \\
\hline 2 & $25.7 \pm 1.1$ & $75.1 \pm 0.8$ \\
\hline 3 & $>100$ & $>100$ \\
\hline 4 & $>100$ & $>100$ \\
\hline 5 & $>100$ & $>100$ \\
\hline 5-fluorouracil ${ }^{b}$ & $2.3 \pm 0.2$ & $1.9 \pm 0.2$ \\
\hline
\end{tabular}

${ }^{a}$ HL-60 and P388 cell lines (each $1 \times 10^{4}$ cells in $100 \mu \mathrm{L}$ ) were treated with test compounds for $72 \mathrm{~h}$, and MTT solution was added to the wells. The grown cells were labeled with $5 \mathrm{mg} / \mathrm{ml}$ MTT in phosphatebuffered saline (PBS), and the absorbance of formazan dissolved with $20 \%$ sodium dodecyl sulfate (SDS) in $0.1 \mathrm{~N} \mathrm{HCl}$ was measured at $550 \mathrm{~nm}$ using a microplate reader. Data are expressed as mean \pm standard deviation (S.D.) $(\mathrm{n}=3){ }^{\mathrm{b}}$ Reference compound. 
Table 3. Melanogenesis inhibitory activities and cytotoxicities in B16 mouse melanoma cell line of multiflorane-type triterpenes isolated from Cucurbita pepo ${ }^{\text {a }}$.

\begin{tabular}{|c|c|c|c|c|c|c|c|c|c|c|c|c|c|c|c|c|c|c|}
\hline \multirow{3}{*}{$\begin{array}{l}\text { Compound } \\
1\end{array}$} & \multicolumn{6}{|c|}{ Mean \pm S.D. (\%) at $10 \mu \mathrm{M}$} & \multicolumn{6}{|c|}{ Mean \pm S.D. (\%) at $30 \mu \mathrm{M}$} & \multicolumn{6}{|c|}{ Mean \pm S.D. (\%) at $100 \mu M$} \\
\hline & \multicolumn{3}{|c|}{ Melanin content } & \multicolumn{3}{|c|}{ Cell viability } & \multicolumn{3}{|c|}{ Melanin content } & \multicolumn{3}{|c|}{ Cell viability } & \multicolumn{3}{|c|}{ Melanin content } & \multicolumn{3}{|c|}{ Cell viability } \\
\hline & 103.7 & \pm & 5.2 & 91.1 & \pm & 4.4 & 99.4 & \pm & 3.7 & 82.3 & \pm & 4.3 & 92.7 & \pm & 3.1 & 76.4 & \pm & 0.8 \\
\hline 2 & 73.6 & \pm & 1.0 & 87.6 & \pm & 0.2 & 69.9 & \pm & 4.4 & 69.3 & \pm & 1.3 & 31.4 & \pm & 2.8 & 32.8 & \pm & 2.8 \\
\hline 3 & 97.3 & \pm & 0.9 & 99.4 & \pm & 4.0 & 93.5 & \pm & 2.5 & 99.4 & \pm & 3.8 & 66.9 & \pm & 5.0 & 92.5 & \pm & 4.3 \\
\hline 4 & 97.4 & \pm & 2.1 & 102.4 & \pm & 4.3 & 96.8 & \pm & 1.0 & 96.2 & \pm & 1.3 & 98.5 & \pm & 8.4 & 88.0 & \pm & 5.9 \\
\hline 5 & 102.0 & \pm & 9.2 & 100.9 & \pm & 1.8 & 101.1 & \pm & 6.9 & 99.2 & \pm & 9.6 & 92.4 & \pm & 4.7 & 97.6 & \pm & 6.6 \\
\hline $\operatorname{arbutin}^{\mathrm{b}}$ & 88.9 & \pm & 2.3 & 100.0 & \pm & 2.7 & 72.3 & \pm & 3.1 & 94.4 & \pm & 1.2 & 55.3 & \pm & 1.0 & 89.9 & \pm & 0.3 \\
\hline
\end{tabular}

${ }^{a}$ Melanin content (\%) and cell viability (\%) were determined based on the absorbances at $450 \mathrm{~nm}$, and $550 \mathrm{~nm}$, respectively, by comparison with those for DMSO (100\%). Each value represents the mean \pm S.D. of three determinations. Concentration of DMSO in the sample solution was $2 \mu \mathrm{L} / \mathrm{mL} ;{ }^{b}$ Reference compound.

\section{Experimental}

\subsection{General Procedures}

Melting points were determined on a Yanagimoto micro-melting point apparatus and are uncorrected. Optical rotations were measured using a JASCO DIP-1000 digital polarimeter. IR spectra were recorded using a Perkin-Elmer $1720 \mathrm{X}$ FTIR spectrophotometer. ${ }^{1} \mathrm{H}$ - and ${ }^{13} \mathrm{C}-\mathrm{NMR}$ spectra were obtained on a Varian INOVA 500 spectrometer with standard pulse sequences, operating at 500 and $125 \mathrm{MHz}$, respectively. $\mathrm{CDCl}_{3}$ was used as the solvent and TMS, as the internal standard. EIMS were recorded on a Hitachi $4000 \mathrm{H}$ double-focusing mass spectrometer (70 eV). Column chromatography was carried out over silica gel (70-230 mesh, Merck, Darmstadt, Germany) and MPLC was carried out with silica gel (230-400 mesh, Merck, Darmstadt, Germany). HPLC was run on a JASCO PU-1586 instrument equipped with a differential refractometer (RI 1531). Fractions obtained from column chromatography were monitored by TLC (silica gel $60 \mathrm{~F}_{254}$, Merck).

\subsection{Plant Material}

The seeds of Cucurbita pepo L. produced in USA (California), were purchased from JA (Japan Agricultural Co-opwration)-Takatsuki in May, 2011.

\subsection{Isolation Procedure}

Air-dried seeds $(10 \mathrm{~kg})$ were ground and extracted $\times 3$ for 3 days each with $\mathrm{MeOH}(10 \mathrm{~L})$ employing an automatic percolator. Removal of the $\mathrm{MeOH}$ under reduced pressure left a greenish residue which was partitioned between $\mathrm{Et}_{2} \mathrm{O}$ and $\mathrm{H}_{2} \mathrm{O}$. Evaporation of the $\mathrm{Et}_{2} \mathrm{O}$ phase gave a yellowish residue $(216.1 \mathrm{~g})$ which was subjected to silica gel $(3.5 \mathrm{~kg})$ column chromatography. Elution of the column with $\mathrm{CHCl}_{3}$ gave residue A (Fr. No. 1-18, 39.5 g), B (Fr. No. 19-25, 14.9 g) and C (Fr. No. 26-30, $10.6 \mathrm{~g})$. Elution of the column with $\mathrm{CHCl}_{3} /$ EtOAc (10:1) afforded residues D (Fr. No. 31-33, $21.5 \mathrm{~g}$ ) and $\mathrm{E}$ (Fr. No. 34-57, $13.4 \mathrm{~g}$ ) and subsequent column chromatography with $\mathrm{CHCl}_{3} / \mathrm{EtOAc}$ (2:1) to give residues F (Fr. (Fr. No. 58-68, 2.0 g). Elution was continued with EtOAc and MeOH to give residues $\mathrm{G}$ (Fr. No. 69-74, $2.0 \mathrm{~g}$ ) and $\mathrm{H}$ (Fr. No. 75-77, $4.5 \mathrm{~g}$ ). 
Residue B was rechromatographed on a silica gel (230-400 mesh, $500 \mathrm{~g})$ column using $n$-hexane:EtOAc $=20: 1 \sim$ EtOAc to give residues B-1 (Fr. Nos. 28-29, 10.9 mg), B-2 (Fr. Nos. 30-33, $30.2 \mathrm{mg}$ ), B-3 (Fr. Nos. 34-39, $32.2 \mathrm{mg}$ ). Residue B-1 was separated by HPLC (Normal phase silica gel, $n$-hexane:EtOAc $=10: 1)$ to give compounds $\mathbf{1}(2.5 \mathrm{mg}), 2(5.1 \mathrm{mg})$ and $\mathbf{3}(1.8 \mathrm{mg})$.

Residue C was rechromatographed on a silica gel (230-400 mesh, $200 \mathrm{~g})$ column using $n$-hexane:EtOAc = 10:1 EtOAc to give residues C-1 (Fr. Nos. 1-20, $176.4 \mathrm{mg}$ ), C-2 (Fr. Nos. 21-39, $60.2 \mathrm{mg}$ ), C-3 (Fr. Nos. 40-47, 3.4 g). Residue C-1-C-3 was separated by HPLC (Normal phase silica gel, $n$-hexane:EtOAc $=10: 1)$ to give compounds $4(28.3 \mathrm{mg})$ and $5(4.9 \mathrm{mg})$. Compound 4 was identified as 3 $\alpha$-p-aminobenzoylmultiflora-7:9(11)-dien-29-benzoate on the basis of published data $[1,2]$, and 5 was identified as $5 \alpha, 9 \alpha$-peroxymultiflora-6,9(11)-diene-3 $\alpha, 29$-dibenzoate on the basis of published data [10].

\subsection{Compound 1}

Colorless crystals; mp $172-174{ }^{\circ} \mathrm{C}$ (from $\left.\mathrm{MeOH}-\mathrm{CHCl}_{3}\right) ;[\alpha]_{\mathrm{D}}{ }^{26}+10.9^{\circ}\left(c 0.048, \mathrm{CHCl}_{3}\right)$; HREIMS $m / z$ : $693.4024[\mathrm{M}]^{+}\left(\mathrm{C}_{44} \mathrm{H}_{55} \mathrm{NO}_{6}\right.$, calcd for 693.4029); UV (EtOH) $\lambda_{\max } \mathrm{nm}(\log \varepsilon): 230$ (3.85), 237 (3.80), 248 (3.63); IR (KBr) $v_{\max } \mathrm{cm}^{-1}$; 2945, 1713 (O-C=O), 1542 (Ar), 1527 and $1341\left(\mathrm{NO}_{2}\right), 1510$, 1371, 1287; ${ }^{1} \mathrm{H}-$ and ${ }^{13} \mathrm{C}-\mathrm{NMR}$, see Table 1. EIMS $\mathrm{m} / z$ (rel. int.): 693 (100) $\left.[\mathrm{M}]^{+}\right), 526$ (41) [M-p-nitrobenzoic acid $]^{+}, 389$ (26), 253 (71), 227 (37), 211 (37).

\subsection{Compound 2}

Colorless crystals; mp 93-94 ${ }^{\circ} \mathrm{C}$ (from $\mathrm{MeOH}-\mathrm{CHCl}_{3}$ ); $[\alpha]_{\mathrm{D}}{ }^{26}-44.0^{\circ}$ (c $0.11, \mathrm{CHCl}_{3}$ ); HREIMS $m / z$ : $586.4016[\mathrm{M}]^{+}\left(\mathrm{C}_{39} \mathrm{H}_{54} \mathrm{O}_{4}\right.$, calcd for 586.4022); UV (EtOH) $\lambda_{\max } \mathrm{nm}(\log \varepsilon): 222$ (3.93), 237 (3.95); IR (KBr) $\nu_{\max } \mathrm{cm}^{-1}$; 2974, 1743, 1718 and 1271 (O-C=O), 1559 (Ar), 1521, 1489, 1458, 1271, 1114; ${ }^{1} \mathrm{H}-$ and ${ }^{13} \mathrm{C}-\mathrm{NMR}$, see Table 1. EIMS $m / z$ (rel. int.): 586 (62) $[\mathrm{M}]^{+}, 526$ (100) [M-HOAc $]^{+}, 511$ (31), 389 (35), 253 (62).

\subsection{Compound 3}

Colorless crystals; mp $105-107{ }^{\circ} \mathrm{C} ;[\alpha]_{\mathrm{D}}{ }^{26}-291.6^{\circ}$ (c 0.255, $\left.\mathrm{CHCl}_{3}\right)$; HREIMS m/z: 584.3864 [M] ${ }^{+}$ $\left(\mathrm{C}_{39} \mathrm{H}_{52} \mathrm{O}_{4}\right.$, calcd for 584.3866); UV $\lambda_{\max }(\mathrm{EtOH}) \mathrm{nm}(\log \varepsilon): 227$ (4.19), 304 (3.98), 315 (4.00), 334 (3.72); IR (KBr) $v_{\max } \mathrm{cm}^{-1}:$ 2949, 2881, 1725 and 1239 (O-C=O), 1540 (Ar), 1450, 1274, 1105, 992, 773; ${ }^{1} \mathrm{H}$ - and ${ }^{13} \mathrm{C}-\mathrm{NMR}$, see Table 1. EIMS $m / z$ (rel. int.): 584 (33) [M] ${ }^{+}, 524$ (100) [M-HOAc] $]^{+}, 509$ (52), 457 (11), 387 (35), 295 (23), 285 (36), 251 (30), 225 (51).

\subsection{Cytotoxicity Assay}

The cytotoxicity assay was determined previously [18]. Briefly, the HL-60 and P388 cell lines (each $1 \times 10^{4}$ cells in $100 \mu \mathrm{L}$ ) were treated with test compounds for $72 \mathrm{~h}$, and MTT solution was added to the wells. The grown cells were labeled with $5 \mathrm{mg} / \mathrm{mL}$ MTT in phosphate-buffered saline (PBS), and the absorbance of formazan dissolved with $20 \%$ sodium dodecyl sulfate (SDS) in $0.1 \mathrm{~N} \mathrm{HCl}$ was measured at $550 \mathrm{~nm}$ using a microplate reader (Model 450, BioRad, Richmond, CA). 


\subsection{Determination of Cell Proliferation}

Cell proliferation was examined according to a method reported previously [19]. Briefly, B16 4A5 cells [obtained from Riken Cell Bank (Tsukuba, Ibaraki, Japan)] $\left(3 \times 10^{4}\right.$ cells in $\left.500 \mu \mathrm{L}\right)$, preincubated for $24 \mathrm{~h}$ were treated for $48 \mathrm{~h}$ with test samples dissolved in dimethyl sulfoxide (DMSO) at a final concentration of 100,30 or $10 \mu \mathrm{M}$, and MTT solution was added. After $3 \mathrm{~h}$ of incubation, 2-propanol containing $0.08 \mathrm{M} \mathrm{HCl}$ was added to dissolve the formazan produced in the cells. The absorbance of each well was read at $550 \mathrm{~nm}$ using a microplate reader.

\subsection{Assay of Melanin Content}

The assay of melanin content was performed as described previously [19]. B16 cells were pre-incubated as above in $\alpha$-MSH $(100 \mathrm{nM})$ containing medium. Test samples dissolved in DMSO were added to the medium and the cells were cultured for $48 \mathrm{~h}$. The medium was removed and the cells were dissolved in $2 \mathrm{M} \mathrm{NaOH}$ containing $10 \%$ DMSO. The amount of melanin was determined spectrophotometrically by measuring absorbance at $450 \mathrm{~nm}$ using a microplate reader. The optical density of control cells was assumed to be $100 \%$.

\section{Conclusions}

The structure of 1 was established as 3 $\alpha$-p-nitrobenzoylmultiflora-7:9(11)-dien-29-benzoate. This is the first report of a triterpenoid having a nitro group in the molecule. At this time we have no explanation for the presence of a $p$-nitrobenzoic moiety in a zucchini metabolite, and wish to consider the role of the nitro group in the plant body in the future.

\section{Supplementary Materials}

Supplementary materials can be accessed at: http://www.mdpi.com/1420-3049/18/7/7448/s1.

\section{Acknowledgments}

We thank Katsuhiko Minoura and Mihoyo Fujitake (this university) for NMR and MS measurements.

\section{Conflict of Interest}

The authors declare no conflict of interest.

\section{References}

1. Appendino, G.; Jakupovic, J.; Belloro, E.; Marchesini, A. Multiflorane triterpenoid esters from pumpkin. An unexpected extrafolic source of PABA. Phytochemistry 1999, 51, 1021-1026.

2. Appendino, G.; Jakupovic, J.; Belloro, E.; Marchesini, A. Triterpenoid $p$-aminobenzoate from the seeds of zucchini. Fitoterapia 2000, 71, 258-263. 
3. Barker, E.C.; Gatbonton-Schwager, T.N.; Han, Y.; Clay, J.E.; Letterio, J.J.; Tochtrop, G.P. Brynolic acid: A large-scale isolation and evaluation of heme oxygenase 1 expression in activated macrophages. J. Nat. Prod. 2010, 73, 1064-1068.

4. Wang, D.; Ge, S.; Gao, H.; Cai, H.; Wu, B.; Li, D.; Wu, L.; Deng, X. Structure determination of a cucurbitacin glycoside extracted from Cucurbita pepo cv Dayangua by 2D NMR. Bopuxue Zazhi 2005, 22, 417-422.

5. Wang, D.; Pan, H.-Y.; Deng, X.-M.; Xiang, H.; Gao, H.; Cai, H.; Wu, L. Cucurbitane and hexanorcucurbitane glycosides from the fruits of Cucurbita pepo cv dayangua. J. Asian Nat. Prod. Res. 2007, 9, 525-529.

6. Wang, D.; Xiang, H.; Li, D.; Gao, H.; Cai, H.; Wu, L.-J.; Deng, X.-M. Purine-containing cucurbitane triterpenoids from Cucurbita pepo $c v$ dayangua. Phytochemistry 2008, 69, $1434-1438$.

7. Ding, Y.; Deng, X.; Cai, Hui.; Wang, F.; Wang, X.; Zhang, Y.; Yang, J. Studies on chemical constituents of Cucurbita pepo cv dayangua. Zhongguo Yaoxue Zazhi 2002, 37, 659-661.

8. Shibuya, M. Biosynthesis of sterols and triterpenes in higher plants. Nat. Med. 2001, 55, 1-6.

9. Shibuya, M.; Adachi, S.; Ebizuka, Y. Cucurbitadienol synthase, the first commited enzyme for cucurbitacin biosynthesis, is a distinct enzyme from cycloartenol synthase for phytosterol biosynthesis. Tetrahedron 2004, 60, 6995-7003.

10. Ma, Y.-P.; Li, N.; Gao, J.; Fu, K.-L.; Qin, Y.; Li, G.-Y.; Wang, J.-H. A new peroxy-multiflorane triterpene ester from the processed seeds of Trichosanthes kirilowii. Helv. Chim. Acta 2011, 94, 1881-1887.

11. Parry, R.; Nishino,S.; Spain, J. Naturally-occurring nitro compounds. Nat. Prod. Rep. 2011, 28, $152-167$.

12. Al-Zereini, W.; Schumann, I.; Laatsch, H.; Helmke, E.; Anke, H. New aromatic nitro compounds from Salegentibacter sp. T436, an Arctic sea ice bacterium: Taxonomy, fermentation, isolation and biological activities. J. Antibiotics 2007, 60, 301-308.

13. Ohmori, T.; Hagiwara, S.-I.; Ueda, A.; Minoda, Y.; Yamada, K. Production of pyoluteorin and its derivatives from n-paraffin by Pseudomonas aeruginosa S10B2. Agric. Biol. Chem. 1978, 42, 2031-2036.

14. Zlatopolskiy, B.D.; Loscha, K.; Alvermann, P.; Kozhushkov, S.I.; Nikolaev, S.V.; Zeeck, A.; de Meijere, A. Final elucidation of the absolute configuration of the signal metabolite hormaomycin. Chem. Eur. J. 2004, 10, 4708-4717.

15. Hoeksema, H.; Mizak, S.A.; Bacznskyj, L.; Pschigoda, L.M. Structure of rubradirin. J. Am. Chem. Soc. 1982, 104, 5173-5181.

16. Alston, T.A.; Mela, L.; Bright, H.J. 3-Nitropropionate, the toxic substance of Indigofera, is a suicide inactivator of succinate dehydrogenase. Proc. Natl. Acad. Sci. USA 1977, 74, 3767-3771.

17. Palermo, J.A.; Rodriguez Brasco, M.F.; Spagnuolo, C.; Seldes, A.M. Illudalane sesquiterpenoids from the soft coral Alcyonium paessleri: The first natural nitrate esters. J. Org. Chem. 2000, 65, $4482-4486$. 
18. Yamada, T.; Muroga, Y.; Jinno, M.; Kajimoto, T.; Usami, Y.; Numata, A.; Tanaka, R. New class azaphilone produced by a marine fish-derived Chaetomium globosum. The stereochemistry and biological activities. Bioorg. Med. Chem. 2011, 19, 4106-4113.

19. Akihisa, T.; Seino, K.; Kaneko, E.; Watanabe, K.; Tochizawa, S.; Fukatsu, M.; Banno, N.; Metori, K.; Kimura, Y. Melanogenesis inhibitory activities of iridoid-, hemiterpene-, and fatty acid-glycosides from the fruits of Morinda citrifolia (Noni). J. Oleo Sci. 2010, 59, 49-57.

Sample Availability: Not available.

(C) 2013 by the authors; licensee MDPI, Basel, Switzerland. This article is an open access article distributed under the terms and conditions of the Creative Commons Attribution license (http://creativecommons.org/licenses/by/3.0/). 